\title{
Overweight and physical inactivity in children living in favelas in the metropolitan region of Recife, Brazil
}

\author{
Excesso de peso e inatividade física em crianças moradoras de favelas na \\ região metropolitana do Recife, $P E$ \\ João Guilherme Bezerra Alves ${ }^{1}$, Pollyanna P. Siqueira², José Natal Figueiroa ${ }^{3}$
}

\section{Resumo}

Objetivo: Verificar a frequência de excesso de peso em crianças de favelas na cidade do Recife, PE, e sua associação com inatividade física.

Métodos: Foi realizado estudo transversal em duas favelas do Recife (Fragoso e Caranguejo), com população total de 9.315 habitantes, sendo 973 crianças na faixa etária compreendida entre 7 e 10 anos, das quais 733 foram incluídas no estudo. Foram consideradas com sobrepeso e obesidade aquelas crianças com índice de massa corporal acima dos percentis 85 e 95 , respectivamente, de acordo com as curvas e as tabelas de percentis do Centro de Controle e Prevenção de Doenças (Centers for Disease Control and Prevention). O nível de atividade física foi avaliado através do questionário de atividade física para crianças, aplicado às crianças na presença das mães. Foram consideradas fisicamente ativas as crianças com escore $\geq 3$ e sedentárias aquelas com escores $<3$.

Resultados: De 733 crianças estudadas, detectou-se excesso de peso em $92(12,6 \%)$ delas. Não houve associação entre excesso de peso e sexo, renda familiar, escolaridade materna, número de irmãos ou horas diárias de assistência à televisão. Entretanto, observou-se maior número de crianças com inatividade física entre aquelas com sobrepeso e obesidade, $66 / 92(71,6 \%)$ versus $363 /$ $641(56,7 \%)(p=0,008)$.

Conclusões: A frequência de excesso de peso entre escolares residentes em favelas na cidade do Recife é elevada e está associada à inatividade física.

\section{Abstract}

Objective: To assess the prevalence of overweight and obesity and its association with physical activity levels in children living in favelas in the city of Recife, state of Pernambuco, Brazil.

Methods: A cross-sectional study was performed with 973 children ranging from 7 to 10 years old, all from two favelas in Recife, Fragoso and Caranguejo (total population of 9,315); of the 973 children assessed, 733 were included in the study. Overweight was defined as body mass index (BMI) equal to or above the 85th percentile, and obesity as BMI equal to or above the 95th percentile, using Centers for Disease Control and Prevention growth charts. Physical activity level was assessed by the Physical Activity Questionnaire for Older Children (PAQ-C). Children with scores $\geq 3$ were considered active, while those with scores $<3$ were considered inactive.

Results: Of the 733 children studied, overweight and obesity were observed in $92(12.6 \%)$. There was no statistical association between physical inactivity and sex, income, maternal schooling, number of siblings and hours of television. However, more children with physical inactivity were observed among overweight or obese children, 66/92 (71.6\%) vs. 363/641 (56.7\%) ( $p=0.008)$.

Conclusions: The prevalence of overweight and obesity is high, and is associated with physical inactivity in children living in favelas in the city of Recife, Brazil. activity.

J Pediatr (Rio J). 2009;85(1):67-71: Child, overweight, obesity, motor

J Pediatr (Rio J). 2009;85(1):67-71: Criança, excesso de peso, obesidade, atividade motora.

1. Doutor. Universidade Federal de Pernambuco (UFPE), Recife, PE.

2. Mestre. Instituto de Medicina Integral Professor Fernando Figueira (IMIP), Recife, PE.

3. Mestre. UFPE, Recife, PE.

Não foram declarados conflitos de interesse associados à publicação deste artigo.

Como citar este artigo: Alves JG, Siqueira PP, Figueiroa JN. Overweight and physical inactivity in children living in favelas in the metropolitan region of Recife, Brazil. J Pediatr (Rio J). 2009;85(1):67-71.

Artigo submetido em 10.09.08, aceito em 28.11.08.

doi:10.2223/JPED.1862 


\section{Introdução}

A obesidade e o sobrepeso vêm apresentando, nas últimas décadas, um importante aumento da prevalência em todo o mundo, especialmente nos países em desenvolvimento que vivenciam uma transição epidemiológica com o melhor controle das doenças infecciosas ${ }^{1-4}$. No Brasil, nos últimos anos, tem se observado um aumento das taxas de excesso de peso entre as populações economicamente menos favorecidas $^{5-7}$. Essas populações sofrem elevados níveis de agravos nutricionais na vida fetal e nos primeiros meses de vida, tendo maiores riscos de desenvolver doença cardiovascular, diabetes melito e obesidade na vida adulta ${ }^{8-10}$. A interação de tais fatores de risco representa um importante e emergente problema de saúde pública para os países em desenvolvimento, pelo aumento da morbimortalidade na população adulta pelas doenças cardiovasculares, principal causa de morte no mundo e também no Brasil, além de elevar de forma importante os custos para o setor da saúde com assistência secundária e terciária dessas afecções ${ }^{11-13}$.

Sendo a obesidade uma afecção metabólica complexa e que resulta, fundamentalmente, do desequilíbrio entre a ingestão e o gasto calórico ${ }^{14,15}$, é importante que se estabeleça qual desses fatores desempenha maior efeito, especialmente entre as populações mais vulneráveis no cenário atual, as crianças que vivem em condições socioeconômicas desfavoráveis. Ainda são desconhecidos nessas populações, em especial entre crianças, os impactos da inatividade física, no novo panorama nutricional do Brasil. Como os hábitos sedentários são habitualmente adquiridos na infância e tendem a permanecer na vida adulta ${ }^{16,17}$, é de fundamental importância a identificação dos hábitos não-saudáveis para que políticas e programas de saúde sejam implantados, visando um melhor controle das doenças crônicas da vida adulta. Dessa forma, foi objetivo do estudo verificar a frequência de sobrepeso/obesidade entre crianças em comunidades de baixa renda e verificar associação com o nível de atividade física.

\section{Métodos}

Estudo transversal realizado nas favelas do Fragoso e Caranguejo, ambas situadas na Região Metropolitana do Recife, estado de Pernambuco. No ano de 2005, essas duas comunidades contavam com uma população total de 9.315 habitantes. O percentual de saneamento básico dessas áreas não ultrapassa $50 \%$, mas cerca de $80 \%$ das construções são de alvenaria e quase $100 \%$ das habitações têm luz elétrica.

Da população total de 973 crianças residentes nas duas favelas, na faixa etária dos 7 aos 10 anos, foram elegíveis 860 delas, por serem cadastradas no Programa Saúde da Família (PSF), desenvolvido pelo Instituto Materno-Infantil Prof. Fernando Figueira (IMIP). Foram excluídas aquelas portadoras de doenças crônicas que afetassem o estado nutricional (cardiopatias, hepatopatias, doenças neurológicas, endócrinas, metabólicas) ou limitassem a locomoção, as portadoras de doenças mentais e aquelas em uso de medicamentos capazes de interferir no peso e/ou estatura. 0 estudo foi realizado no período de janeiro a agosto de 2006.

O peso foi aferido, sempre na unidade básica de saúde, através de balança digital, previamente calibrada, da marca Filizola $^{\mathrm{TM}}$, com capacidade de até $180 \mathrm{~kg}$ e graduação a cada $100 \mathrm{~g}$. A criança estava sempre sem calçados e apenas com as roupas de baixo. A estatura foi mensurada com as crianças na posição em pé, descalças, com um estadiômetro vertical da marca Stanley (precisão $0,1 \mathrm{~cm}$ ). O índice de massa corporal foi calculado como peso em $\mathrm{kg}$ dividido pela estatura em $\mathrm{m}^{2}$. Foram consideradas com excesso de peso aquelas crianças com o índice de massa corporal acima do percentil 85 para o sexo e idade e obesas com o índice de massa corporal acima do percentil 95, de acordo com as curvas e as tabelas de percentis do índice de massa corporal do Centers for Disease Control and Prevention (CDC) ${ }^{18}$.

O nível de atividade física foi avaliado através do questionário de atividade física para crianças (Physical Activity Questionnaire for Older Children, $\mathrm{PAQ}-\mathrm{C})^{19}$, aplicado às crianças na presença das mães. Esse questionário foi traduzido para o português e validado por da Silva \& Malina ${ }^{20}$ e com exclusão de atividades físicas e esportivas não praticadas no Brasil. Tal questionário avalia o nível de atividade física moderada e intensa de crianças nos 7 dias anteriores à aplicação do mesmo. O questionário é composto de nove questões sobre a prática de esportes e jogos e atividades físicas na escola e no tempo de lazer, incluindo o final de semana. Cada questão tem valor de 1 a 5 e o escore final é obtido pela média das questões, representando o intervalo de muito sedentário (1) a muito ativo (5). Os escores 2, 3 e 4 indicam as categorias sedentário, moderadamente ativo e ativo, respectivamente. Sendo assim, a partir do escore podem-se classificar os indivíduos como ativos ou sedentários. Ativos são aqueles que têm escore $\geq 3$, enquanto sedentários são os indivíduos com escores $<3$. O questionário avalia também o número diário de horas gastas assistindo a televisão.

Os dados coletados foram digitados com dupla entrada e registrados no Programa Epi-Info versão 6. A análise estatística baseou-se na aplicação do teste do qui-quadrado de associação de Pearson para analisar a associação entre as variáveis e o desfecho. O nível de significância foi estabelecido em 0,05 e as análises foram executadas pelo programa Statistical Package for the Social Science (SPSS), versão 8.0.0 (SPSS Incorporation,1977).

O projeto do estudo foi previamente aprovado pelo Comitê de Ética e Pesquisa do IMIP e seguiu as orientações da Resolução CNS no 196/1996. Todos os pais das crianças participantes assinaram o termo de consentimento esclarecido. 
Tabela 1 - Distribuição das variáveis biológicas e socioeconômicas das crianças estudadas com e sem excesso de peso/obesidade em favelas da região metropolitana do Recife (PE)

\begin{tabular}{|c|c|c|c|}
\hline \multirow[b]{2}{*}{ Variável } & \multicolumn{2}{|c|}{ Sobrepeso/obesidade } & \multirow[b]{2}{*}{$\mathbf{p}$} \\
\hline & $\begin{array}{c}\operatorname{Sim}(n=92) \\
n(\%)\end{array}$ & $\begin{array}{c}\text { Não }(n=641) \\
n(\%)\end{array}$ & \\
\hline Sexo masculino & $41(44,5)$ & $267(41,7)$ & 0,677 \\
\hline Renda familiar $<1$ SM & $60(65,0)$ & $432(67,5)$ & 0,766 \\
\hline Geladeira em casa & $57(61,9)$ & $423(65,9)$ & 0,519 \\
\hline Televisão em casa & $85(92,3)$ & $607(94,6)$ & 0,511 \\
\hline Escolaridade materna $<4$ anos & $28(30,3)$ & $184(28,7)$ & 0,826 \\
\hline Número de irmãos $>2$ & $44(47,5)$ & $282(44,0)$ & 0,562 \\
\hline Assiste a televisão $>3 \mathrm{~h} / \mathrm{dia}$ & $68(74,0)$ & $459(71,5)$ & 0,736 \\
\hline Escore PAQ-C $<3$ & $66(71,6)$ & $363(56,7)$ & 0,008 \\
\hline
\end{tabular}

PAQ-C = questionário de atividade física para crianças (Physical Activity Questionnaire for Older Children); SM = salário mínimo.

\section{Resultados}

Das 860 crianças elegíveis, foram estudadas 733 $(85,2 \%) ; 122$ crianças não foram localizadas ou não compareceram à unidade de saúde para a coleta dos dados e cinco apresentaram morbidades que preenchiam os critérios de exclusão. Em $92(12,6 \%)$ crianças foi detectado sobrepeso $(73,10 \%)$ ou obesidade $(19,2,6 \%)$, sendo $44,5 \%$ meninos e $55,5 \%$ meninas.

Não houve associação estatística significante entre sexo, renda familiar, geladeira ou televisão no domicílio, escolaridade materna, número de irmãos ou horas diárias assistindo a televisão. Entretanto, observou-se um maior número de crianças com inatividade física, escore do PAQ-C $<3$, entre as crianças com sobrepeso e obesidade (Tabela 1 ).

\section{Discussão}

São escassos os estudos realizados em favelas, no Brasil, com o objetivo de determinar a frequência de sobrepeso/ obesidade em crianças. Estudo publicado na década passada, realizado em uma favela na cidade de São Paulo (SP), encontrou prevalência de excesso de peso de $6,4 \%$ em meninos e de $8,7 \%$ em meninas ${ }^{21}$. Como o problema do sobrepeso e da obesidade vem aumentando de forma importante no Brasil, especialmente nas camadas mais pobres, pode ser que tal percentual, observado em São Paulo há mais de 10 anos, seja hoje semelhante ao encontrado em nosso estudo no Recife. Outros estudos realizados em favelas no Brasil apontam frequência de excesso de peso e obesidade entre a população geral adulta de $15,7 \%$ de sobrepeso/obesidade, sendo essa prevalência de $22,7 \%$ entre as mulheres ${ }^{22}$.Quando comparamos nossos resultados com estudos realizados em crianças de boas condições socioeconômicas, em várias regiões do Brasil, observamos uma prevalência de obesidade quase duas vezes maior entre essas crianças de melhores condições socioeconômicas ${ }^{23-25}$. Entretanto, estudamos crianças que vivem em constante risco de insegurança alimentar, devido à renda familiar ser muito baixa. Admitimos que a justificativa para tais achados encontre-se no custo dos alimentos, pois essa população tem acesso a alimentos de custo mais baixo, habitualmente com elevado teor de açúcar e gordura. A relação do excesso de peso com os padrões socioeconômicos muitas vezes é inconsistente e não guarda um padrão definido, não podendo, assim, ser generalizada ${ }^{6}$.

Observamos elevado número de crianças fisicamente inativas. Uma justificativa em particular para tal achado parece ser a falta de espaço para o desenvolvimento de atividades físicas. As casas não têm quintais, e em alguns locais faltam ruas, sendo que a maioria tem largura de pouco mais de $1 \mathrm{~m}$. Registre-se ainda a inexistência de locais amplos ou praças, onde pudessem ser desenvolvidas atividades físicas. Quando comparamos a inatividade física, segundo o PAQ-C, entre as crianças com e sem excesso de peso/obesidade, verificamos maior nível de atividade física entre as crianças eutróficas. Entretanto, por ter se tratado de estudo de prevalência, não podemos assegurar uma relação de causa e efeito entre essas duas variáveis. Crianças com excesso de peso ou obesidade podem apresentar maiores dificuldades de locomoção, na prática de esportes e em brincadeiras que exijam atividade física mais vigorosa ${ }^{26}$.

O hábito de assistir a televisão por mais de 3 horas diárias foi observado na maioria das crianças, não havendo diferença naquelas portadoras ou não de excesso de peso/ 
obesidade. Vários estudos têm apontado uma relação direta entre número de horas gastas assistindo a televisão e obesidade $^{26,27}$. Pode ser que um dos papéis desempenhados pela televisão para o aumento da obesidade, o estímulo ao consumo alimentar pela propaganda, não possa ser atendido por essa população devido ao baixo poder aquisitivo. Registre-se a presença de aparelhos de televisão nos domicílios em uma frequência maior do que a de geladeiras.

Para alguns autores, o simples consumo alimentar não justifica o aumento da obesidade em populações de baixo poder aquisitivo ${ }^{28}$. Algumas pesquisas têm apontado que crianças com subnutrição nos primórdios da vida, apresentam maior risco de tornar-se adultos com excesso de peso e, particularmente, desenvolver obesidade visceral ${ }^{29,30}$. Tais estudos apontaram para um menor gasto energético dessas crianças, alterações no mecanismo de oxidação de lipídeos e maior velocidade de crescimento, todos mecanismos compensatórios, imediatos e tardios, frente aos agravos nutricionais ${ }^{30,31}$.

Temos algumas limitações em nosso estudo. Primeiro, o questionário utilizado tem a limitação de não discriminar intensidade, frequência e duração das atividades físicas e não estimar o gasto calórico ${ }^{19}$. Segundo, esse questionário foi descrito para o uso em crianças entre 8 e 12 anos de idade e nós o utilizamos em crianças de 7 anos, muito embora tenhamos aplicado os questionários com a presença das mães. Também devemos registrar a impossibilidade do desenho de estudo utilizado de responder se a maior frequência de inatividade física, observada em crianças com excesso de peso foi a causa ou a consequência desse desvio nutricional.

Em conclusão, é elevada a prevalência de excesso de peso em crianças da faixa etária escolar, residentes em favelas na região metropolitana do Recife, o que implica em aumento de tal distúrbio nutricional na vida adulta, elevando as chances de doenças crônicas degenerativas para essa população. $O$ sobrepeso e a obesidade estão associados à inatividade física dessas crianças, havendo necessidade de novos estudos com tais populações para melhor compreensão dessa associação e a implantação de políticas de saúde pública para seu controle.

\section{Referências}

1. Campbell T, Campbell A. Emerging disease burdens and the poor in cities of the developing world. J Urban Health. 2007;84:i54-64.

2. Popkin BM. Global nutrition dynamics: the world is shifting rapidly toward a diet linked with noncommunicable diseases. Am J Clin Nutr. 2006;84:289-98.

3. Prentice AM. The emerging epidemic of obesity in developing countries. Int J Epidemiol. 2006;35:93-9.

4. Rivera JA, Barquera S, González-Cossio T, Olaiz G, Sepúlveda J. Nutrition transition in Mexico and in other Latin American countries. Nutr Rev. 2004: 62:S149-57.
5. Batista Filho M, Rissin A. A transição nutricional no Brasil: tendências regionais e temporais. Cad Saude Publica. 2003; 19 Suppl 1: S181-91.

6. Monteiro CA, Conde WL, Popkin BM. The burden of disease from undernutrition and overnutrition in countries undergoing rapid nutrition transition: a view from Brazil. Am J Public Health. 2004; 94:433-4.

7. Sichieri R, Nascimento S, Coutinho W. The burden of hospitalization due to overweight and obesity in Brazil. Cad Saude Publica. 2007;23:1721-27.

8. Barker DJ. The developmental origins of adult disease. Eur J Epidemiol. 2003;18:733-6.

9. Gluckman PD, Hanson MA, Pinal C. The developmental origins of adult disease. Matern Child Nutr. 2005;1:130-41.

10. Alves JG, Figueira F. Doenças do adulto com raízes na infância. Recife: Bagaço; 1998.

11. Krishnaswamy K, Naidu AN, Prasad MP, Reddy GA. Fetal malnutrition and adult chronic disease. Nutr Rev. 2002; 60:S35-9.

12. Alves JG, Figueiroa JN. Infant mortality in Brazil and deaths from acute myocardial infarction in the same generation. Cad Saude Publica. $2004 ; 20: 1525-30$.

13. Alves JG. Low birth weight and early weaning: new risk factors for atherosclerosis. J Pediatr (Rio J). 2004;80:339-40.

14. Gesta S, Tseng YH, Kahn CR. Developmental origin of fat: tracking obesity to its source. Cell. 2007;131:242-56.

15. Kokkinos P, Moutsatsos G. Obesity and cardiovascular disease: the role of diet and physical activity. J Cardiopulm Rehabil. 2004; 24:197-203

16. Guo SS, Chumlea WC. Tracking of body mass index in children in relation to overweight in adulthood. Am J Clin Nutr. 1999; $70: 145 S-8 S$.

17. Alves JG, Montenegro FM, Oliveira FA. Prática de esportes durante a adolescência e atividade física de lazer na vida adulta. Rev Bras Med Esporte. 2005; 11:291-4.

18. National Center for Health Statistics. New pediatric growth charts provide tool to ward off future weight problems. HHS News. May 30, 2000. http://www.cdc.gov/nchs/pressroom/00news/ growchrt.htm. Acesso: 12/01/2009.

19. Crocker PR, Bailey DA, Faulkner RA, Kowalski KC, McGrath R. Measuring general levels of physical activity: preliminary evidence for the Physical Activity Questionnaire for Older Children. Med Sci Sports Exerc. 1997;29:1344-9.

20. da Silva RC, Malina RM. Nível de atividade física em adolescentes do Município de Niterói, Rio de Janeiro. Cad Saude Publica. 2000; 16:1091-7.

21. Sawaya AL, Dallal G, Solymos G, de Sousa MH, Ventura ML, Roberts $S B$, et al. Obesity and malnutrition in a shantytown population in the city of São Paulo, Brazil. Obes Res 1995; 3 Suppl 2:107s-15s.

22. Suñé FR, Dias-da-Costa JS, Olinto MT, Pattussi MP. Prevalence of overweight and obesity and associated factors among schoolchildren in a southern Brazilian city. Cad Saude Publica. 2007;23:1361-71.

23. Costa RF, Cintra Ide P, Fisberg M. Prevalence of overweight and obesity in school children of Santos city, Brazil. Arq Bras Endocrinol Metabol. 2006;50:60-7. 
24. Abrantes MM, Lamounier JA, Colosimo EA. Overweight and obesity prevalence among children and adolescents from Northeast and Southeast regions of Brazil. J Pediatr (Rio J). 2002; 78:335-40.

25. Florêncio TT, Ferreira HS, Cavalcante JC, Stux GR, Sawaya AL. Short stature, abdominal obesity, insulin resistance and alterations in lipid profile in very low-income women living in Maceió, north-eastern Brazil. Eur J Cardiovasc Prev Rehabil. 2007;14:346-8.

26. Reilly J J, McDowell ZC. Physical activity interventions in the prevention and treatment of paediatric obesity: Systematic review and critical appraisal. Proc Nutr Soc. 2003;62:611-9.

27. Caroli M, Argentieri L, Cardone M, Masi A. Role of television in childhood obesity prevention. Int J Obes Relat Metab Disord. 2004;28 Suppl 3:S104-8.

28. Florêncio TT, Ferreira HS, Cavalcante JC, Luciano SM, Sawaya AL. Food consumed does not account for the higher prevalence of obesity among stunted adults in a very-low-income population in the Northeast of Brazil (Maceió, Alagoas). Eur J Clin Nutr. 2003; 57:1437-46.
29. Hoffman DJ, Sawaya AL, Coward WA, Wright A, Martins PA, de Nascimento C, Tucker KL, Roberts SB. Energy expenditure of stunted and nonstunted boys and girls living in the shantytowns of São Paulo, Brazil. Am J Cin Nutr. 2000;72:1025-31.

30. Hoffman DJ, Sawaya AL, Verreschi I, Tucker KL, Roberts SB. Why are nutritionally stunted children at increased risk of obesity? Studies of metabolic rate and fat oxidation in shantytown children from São Paulo, Brazil. Am J Clin Nutr. 2000;72:702-7.

31. Grillol LP, Siqueira AF, Silva AC, Martins PA, Verreschi IT, Sawaya AL. Lower resting metabolic rate and higher velocity of weight gain in a prospective study of stunted vs nonstunted girls living in the shantytowns of São Paulo, Brazil. Eur J Clin Nutr. 2005;59:835-42.

Correspondência:

João Guilherme Bezerra Alves

Rua dos Coelhos, 300

CEP 50070-550 - Recife, PE

Tel.: (81) 2122.4122

Tel.: (81) 9974.6351

E-mail: joaoguilherme@imip.org.br 